\title{
Comprehension of the Communicative Intent Behind Pointing and Gazing Gestures by Young Children with Williams Syndrome or Down Syndrome
}

\author{
Angela E. John and Carolyn B. Mervis \\ University of Louisville
}

\begin{abstract}
Purpose-This study examined the ability of preschoolers with Williams syndrome (WS) or Down syndrome (DS) to infer communicative intent as expressed through gestures (pointing and eye gaze shift).
\end{abstract}

\begin{abstract}
Methods-Participants were given a communicative or non-communicative cue involving pointing or gaze shifting in the context of a hiding game. Each child completed four conditions formed by crossing Communicative style (communicative vs. non-communicative) and Gesture (point vs. gaze shift).
\end{abstract}

Results-At the group level, children in both groups located the toy significantly more often than expected by chance in the communicative condition but performed at chance in the noncommunicative condition. Children in both groups were more likely to infer communicative intent when pointing rather than gaze shifting was used. Individually, despite significantly lower DQ and language standard scores, significantly more children with DS than with WS successfully used the experimenter's communicative gestures.

Conclusions-At the group level, preschoolers with WS or DS were able to comprehend the communicative intent expressed by pointing and gazing gestures in a table-top task. Children with DS evidenced significantly stronger pragmatic skills than did children with WS, providing further evidence that children with WS have more difficulty with socio-communication than expected for CA or cognitive/language ability.

\section{Keywords}

Williams-Beuren syndrome; Down syndrome; communicative intent; social communication; pragmatics; gesture; intellectual disability

\footnotetext{
The development of intentional communication is important for children's effective functioning in the social world as it allows them to predict, explain, and even manipulate people's actions, giving children the ability both to understand others and to make themselves understood (Akhtar \& Martinez-Sussmann, 2007; Hala, 1997). Although typically developing (TD) children develop a basic understanding of the psychological world of people at an early age (see Thompson, 2006 for review), this is no easy task. Interactions with people are far more complex than interactions with objects because human behavior stems from underlying mental states (e.g., goals, intentions, feelings, desires, thoughts, and beliefs; Thompson, 2006). Meltzoff (1995) pointed out that human beings
}

Address Correspondence to: Angela E. John, Dept. of Psychological and Brain Sciences, University of Louisville, Louisville, KY 40292. Telephone: 502-852-4638, Fax: 502-852-8271, aejohn11@ gwise.louisville.edu. 
would be very difficult to predict, and even harder to explain, if we had to restrict our understanding of them to their physical behaviors and movements. As such, it is clear how important the ability to understand that there are intentions and goals behind people's actions and gestures is for social cognitive development; this understanding allows a child to interpret why someone is doing what he/she is doing, as well as to recognize the significance of these actions (Meltzoff, 1995).

By 12 months of age, most TD infants have entered an intentional (illocutionary) stage in which they demonstrate both control over their own communicative behaviors and the ability to monitor and comprehend the communicative behavior of others (e.g., Akhtar \& Martinez-Sussmann, 2007; Bates, Camaioni, \& Volterra, 1975; Carpenter, Nagell, \& Tomasello, 1998; Moore \& Corkum, 1994). For example, around this age, TD children are able to follow another person's gazing gesture (head turn paired with eye gaze shift; e.g., Brooks \& Meltzoff, 2002; Butterworth, 2001; Corkum \& Moore, 1995; Moore \& Corkum, 1994) and can reliably follow a pointing gesture to a distal target (e.g., Akhtar \& MartinezSussmann, 2007; Butterworth, 2003; Carpenter, et al., 1998; Tomasello, Carpenter, \& Liszkowski, 2007). However, as discussed by Behne, Carpenter, and Tomasello (2005), the mere act of following a pointing or gazing gesture does not necessarily indicate that the child comprehends the communicative intent underlying these actions. To demonstrate comprehension of communicative intent, one must not only show that the child follows the gesture to an otherwise uninteresting object, but also that the child makes an inference about why the adult directed the child's attention (Behne, Carpenter, \& Tomasello, 2005). To begin to address this issue, researchers have started to examine TD children's comprehension of the communicative intent behind gestures. In comparison, there have been only a few studies of gestural comprehension by children with genetic disorders and none of these studies has directly considered the question of comprehension of communicative intent.

The purpose of the present study was to examine the ability of preschoolers who have Williams syndrome (WS) or Down syndrome (DS) to comprehend the communicative intent behind pointing and gazing gestures. These syndromes were chosen both because they are well defined genetically and because they are characterized by different patterns of social communicative ability relative to overall intellectual ability. In the remainder of the introduction, we provide short descriptions of WS and DS and then briefly review prior studies of TD children's comprehension of the communicative intent behind nonlinguistic gestures and the ability of children with WS or DS to follow nonlinguistic gestures.

WS is a neurodevelopmental disorder resulting from a hemideletion of $\sim 25$ geneson chromosome 7q11.23 (Ewart et al., 1993; Morris, 2006), with a prevalence of 1 in 7500 live births (Strømme, Bjørnstad, \& Ramstad, 2002). WS is characterized by mild to moderate intellectual disability or learning difficulties, dysmorphic facial features, and heart disease, especially supravalvar aortic stenosis (Morris, 2006). Individuals with WS demonstrate relative strengths in concrete vocabulary and verbal short-term memory (Mervis \& KleinTasman, 2000; Mervis \& Morris, 2007; Udwin \& Yule, 1990). Children with WS are often described as friendly, outgoing, and never going unnoticed in a group (Dilts, Morris, \& Leonard, 1990; Dykens \& Rosner, 1999; Fryns, Borghgraef, Volcke, \& van den Berge, 1991; Gosch \& Pankau, 1997). Despite these positive characteristics, children with WS demonstrate significant difficulties interacting with others and significant impairments in pragmatic abilities (for review see Mervis \& Becerra, 2007; Tager-Flusberg \& Plesa Skwerer, 2006). The nonverbal communicative behaviors of toddlers and preschoolers with WS (e.g., gaze shifting, participation in joint attention, production of gestures, tracking distal pointing gestures) are well below both chronological age (CA) and mental age (MA) expectations (Laing et al., 2002; Rowe, Peregrine, \& Mervis, 2005). 
DS is a more common neurodevelopmental disorder (1 in 733 live births; Centers for Disease Control, 2006), resulting from the presence of an extra copy of the long arm of chromosome 21. This disorder is also associated with a particular pattern of dysmorphic facial characteristics, heart defects, and intellectual disability. In addition, DS is characterized by IQ scores that are slightly but significantly lower than in WS (Edgin, 2003; Klein \& Mervis, 1999; Rowe, 2007), relative strength in nonverbal communication (Miller \& Leddy, 1999), and a weakness in expressive language (Chapman, 1999). Social emotional skills have been described as a relative strength for children with DS (Fidler, 2005; Kasari, 2002), and children with DS demonstrate competence in forming relationships with others (Kasari, Freeman, \& Bass, 2003), potentially relying on their social strengths to compensate for their other limitations.

\section{Comprehension of Communicative Intent Behind Gestures: Typical Development}

The ability to follow another person's gazing gesture (head turn paired with eye gaze shift) or pointing gesture is an important milestone in early communicative development as it serves as a governing factor in both social interactions and referential communication between a young child and an adult (Adamson, 1995; D'Entremont, Hains, \& Muir, 1997). Recently researchers have narrowed their examination of young TD children's ability to follow another's gesture, focusing on their ability to comprehend the communicative intent behind the gestures. For example, Tomasello, Call, and Gluckman (1997) conducted a study in which one adult hid a reward in one of three different-looking containers while a second adult indicated the location of the reward to the child using one of three methods: (1) pointing to the correct container; (2) placing a small wooden block on the correct container; or (3) showing the child an exact replica of the container in which the reward was hidden. The authors then examined the ability of three groups of participants $\left(2 \frac{1}{2}\right.$-year-old TD children, 3-year-old TD children, and great apes) to interpret these cues as an indication of the location of the reward. Results indicated that both groups of children not only followed the adult's indication to one of the containers at a rate significantly above chance but also interpreted the adult's behavior as an indication of the reward's location, as they looked in the specified container for all three cues. Both groups were most likely to find the reward in the pointing condition, next most likely to find the reward in the wooden-block condition, and least likely to find the reward in the exact-replica condition. In contrast, while the great apes understood the nature of the hiding game and searched for the reward they did not locate the hidden reward at a rate significantly above chance, indicating an inability to infer the communicative intent behind the cue.

Recently, Behne et al. (2005) examined the ability of TD 14-, 18-, and 24-month-olds to comprehend the communicative intent behind pointing and gazing gestures. In their first study, the experimenter hid a toy in one of two identical containers, established eye contact with the child, and provided a communicative cue (point or head/gaze shift paired with communicative intent expressed through facial expressions). The ability to reliably identify the correct location of the hidden toy was theorized to signify that the child used the adult's communicative cues to find the toy. Results demonstrated that at the group level TD children of all three ages were able to find the hidden toy significantly more often than would have been expected by chance. At the individual child level, search performance improved significantly with age: $7 \%$ of 14-month-olds, $60 \%$ of 18 -month-olds, and $84 \%$ of 24-month-olds found the toy significantly more often than expected by chance.

To control for the possibility that children's success in that study was influenced by the mere act of extending an index finger or moving one's eyes from one location to another and was not influenced by the adult's intent, the authors repeated the study with additional groups of 
14-and 18-month-olds with one important change. This time, the cue presented by the experimenter had the same surface behavior but was non-communicative (point while examining her hand or absent minded-head/gaze shift with chin resting on hand). Results indicated that the performance of both groups was not significantly above chance. Behne and colleagues (2005) interpret their findings as indicating that by 14 months of age children understand that some actions by adults express communicative intent and that by 24 months of age TD children reliably attend to pointing and gazing gestures when used communicatively, recognizing that the behavior is intended to communicate something to them and therefore is relevant to their ongoing interaction.

\section{Comprehension of Communicative Gestures: WS and DS}

To date, only a few studies have examined the ability of children with WS or DS to follow pointing or gazing gestures. Two studies (Klein-Tasman, Mervis, Lord, \& Phillips, 2007; Lincoln, Searcy, Jones, \& Lord, 2007) reported on the ability of children with WS of similar age $(30-63$ months vs. $27-58$ months) to follow a gazing or pointing gesture during the Autism Diagnostic Observation Schedule - Generic (ADOS-G; Lord, Rutter, DiLavore, \& Risi, 1999) Module 1. Klein-Tasman et al. (2007) found that $34 \%$ of the children in their sample were unable to use an experimenter's communicative gaze shift to locate a target from a distance. Half of these children (17\% of the total sample) were still unable to locate the intended target even when a communicative pointing gesture was used. Lincoln et al (2007) reported that $20 \%$ of their sample had difficulty following a communicative eye-gaze gesture; no information was provided regarding the percentage of children demonstrating difficulty using the experimenter's eye gaze paired with a pointing gesture.

Laing et al. (2002) examined the early communicative behaviors of 13 children with WS aged $17-55$ months (mean= 31months) compared to13 TD children(mean $\mathrm{CA}=13$ months)whose CA matched the children with WS's MA. Using the Early Social Communication Scales (ESCS; Mundy \& Hogan, 1996), a semi-structured play based assessment designed to press for early communicative behaviors, Laing et al. found that the WS group followed the adult's pointing gestures (whether proximal or distal) less often than the MA-matched TD group (30.8\% vs. $41.06 \%$ of the time). However, this difference did not reach conventional significance levels $(p=.07$ vs. $p<.05)$. The authors also reported data for a second study using 11 of the MA-matched pairs (Mean CA: WS $=29.6$ months vs. $\mathrm{TD}=13.1$ months) examining the ability to follow an adult's point to distal objects. Results indicated that the children with WS followed the adult's point less often than the TD children did $(p=.05)$. Using a similar task, Rowe, Peregrine, and Mervis (2005) compared the performance of 10 pairs of children (one child with WS, one child with DS) matched on CA (range $18-26$ months; $p=.56$ ), developmental quotient (DQ; $p=.76$ ), and expressive vocabulary size $(p=.36)$. Results indicated that the toddlers with DS successfully followed a distal pointing gesture significantly more often than did the toddlers with WS $(p=.008)$.

Several studies examining responding to joint attention bids have included children with DS. However, in almost all cases, the participants with DS were either adolescents (e.g.,

Attwood, Frith, \& Hermelin, 1988) or young children included in a mixed etiology group for which findings specific to the DS subgroup were not reported. Only one other study has addressed gesture following by young children with DS. Sigman and Ruskin (1999) examined social competence in MA-matched groups of children with autism (CA $3-11$ years), children with DS (CA 2 - 8 years), children with developmental delays (CA 2 - 6 years), and TD children (mean CA 19.49 months). The authors reported that the DS group was significantly more likely than the autism group to follow another person's gazing gesture. The performance of the DS group did not differ significantly from that of the developmental delay or TD groups. Point following was not assessed. 


\section{Present Study}

The results of the studies just described indicate that some toddlers and preschoolers with WS or DS are able to follow pointing or gazing gestures. Consistent with prior findings that children with WS have socio-communicative difficulties beyond those expected for their overall developmental level whereas children with DS show a relative strength in sociocommunication, the results of one study indicated that children with DS are better at following pointing gestures than are matched children with WS. However, none of these studies addressed the question of whether children with WS or DS comprehended their partner's communicative intent. In the present study, we used a modified version of Behne et al.'s (2005) communicative intent task to examine the ability of preschoolers with WS or DS to recognize communicative intent as expressed through non-linguistic gestures (eye gaze shift or pointing) in the context of a hiding game. The same cues were presented to the children both in a communicative and a non-communicative manner to more clearly examine if the children comprehended their partner's communicative intent when retrieving a hidden toy and to evaluate the possibility that the sole act of extending a index finger or shifting one's head and eyes between the child and the correct location, regardless of the partner's communicative intent, influenced children's search performance.

\section{METHOD}

\section{Participants}

Participants were 33 children with genetically-confirmed WS ( 17 boys, 16 girls) aged 3.00 to 5.33 years $(M=4.14, S D=0.68)$ and 25 children with genetically-confirmed DS (11 boys, 14 girls) aged 3.02 to 5.40 years $(M=4.34, S D=0.73)$. All children with WS had classic-length deletions and all children with DS had trisomy 21. Six additional children (4 with WS, 2 with DS) were excluded because of comorbid diagnoses on the autism spectrum based upon ADOS-G, ADI-R, and clinical impression. Administration of the task was discontinued for an additional 6 children (4 with WS, 2 with DS) because they were unwilling to search for the toy on any of the warm up trials. The remaining children searched for the hidden toy on all trials in both the communicative and non-communicative conditions. The participating families varied in terms of their ethnic and racial backgrounds. In the WS group, 82\% were Caucasian, 6\% African American, 6\% Hispanic, and 6\% Asian. In the DS group, $88 \%$ were Caucasian, $8 \%$ African American, and $4 \%$ Hispanic.

\section{Standardized Assessment}

Mullen Scales of Early Learning (Mullen, 1995). The Mullen is an individually administered standardized measure of cognitive functioning for children aged 1-68 months. The Mullen provides a composite DQ standard score (similar to IQ) based on performance on four scales: Visual Reception, which primarily assesses visual perceptual, memory, and nonverbal conceptual ability; Fine Motor, which assesses primarily visuospatial construction; Receptive Language; and Expressive Language, which assesses expressive language and verbal short-term memory.

\section{Procedure}

The Mullen was administered according to standardized procedures, within a day of the communicative intent task.

Communicative Intent Task (Modeled after Behne et al., 2005)—This task was designed to press for the child's ability to infer communicative intent as expressed through both pointing and gazing gestures and was presented in the context of a hiding game. Four warm-up trials were given to familiarize the child with the boxes used for the hiding game. 
The child was shown a pair of identical empty containers and an attractive toy. Experimenter 1 then hid the toy in one of the containers as the child watched. After the containers were closed, the child was asked, "Where is the [toy name]?" This "visible hiding" procedure was carried out for all four warm-up trials. When the hidden toy was located the experimenter excitedly responded saying, "There it is!" Testing was discontinued if the child did not search for the toy on the warm-up trials.

For the experimental trials ( 24 trials total), the experimenter knelt behind a $48^{\prime \prime} \times 22^{\prime \prime}$ opaque barrier that was placed on the floor behind a low table $\left(35^{\prime \prime} \times 19.5^{\prime \prime} \times 6.5^{\prime \prime}\right)$ facing the child and an assistant. At the start of the trial, a pair of open, empty containers was placed on the table, one at each end. The experimenter showed the child a toy of interest and told the child that she was going to hide it. The experimenter then moved the boxes behind the barrier and placed the toy in one of the boxes out of sight of the child, so that he or she did not know which box contained the toy. The experimenter then put the boxes back on the table, one at each end so that they were equidistant from both the experimenter and the child. This was done to ensure that the child could not open both boxes at the same time so would have to choose which one to approach. Once the boxes were positioned on the table, the experimenter first established eye contact with the child and then provided either a noncommunicative or communicative cue (Communicative style) using pointing or gaze shifting (Gesture). The assistant sat with the child to ensure the child stayed positioned in the center of the table equidistant to the two containers until the experimenter demonstrated the specified cue. If the child had difficulty opening a container, the assistant aided the child. The assistant was blind to the hypotheses concerning the study.

Each child participated in four conditions (each including 6 trials) formed by crossing Communicative style (non-communicative vs. communicative) and Gesture type (eye gaze shift + point vs. eye gaze shift only). All of the cues provided were non-verbal. Two different experimenters were used to administer this task. Experimenter1 administered the warm-up trials and the non-communicative trials and Experimenter 2 administered the Communicative trials. Both experimenters established eye contact with the child prior to providing their cue. Once the cue was demonstrated, both experimenters asked the child," Where is the [toy name]?", encouraging the child to search for the hidden toy. The four types of non-verbal cues were:

a. Non-communicative point+ gaze: After establishing eye contact with the child, the experimenter looked down at her hand, which was held at a point at her midline directed toward the box where the toy was hidden. The experimenter's hand was equidistant from both boxes. The experimenter's facial expression demonstrated that she was preoccupied with inspecting something on her wrist on the hand held in a point (a bracelet when pointing with her right hand toward the box on the left; a watch when pointing with her left hand toward the box on the right).

b. Non-communicative gaze: After establishing eye contact with the child, the experimenter rested her chin in her hand and shifted her gaze between the baited container and the direction in which the child was sitting twice while demonstrating an absent-minded (bored) non-communicative expression.

c. Communicative point+ gaze: After establishing eye contact with the child, the experimenter demonstrated a point (held at her midline) across her body at the box in which the toy was hidden. In addition, the experimenter shifted her gaze between the baited container and the child twice, expressing her communicative intent through facial expressions (e.g., raised eyebrows and smile).

d. Communicative gaze: After establishing eye contact with the child the experimenter turned her head, gazing between the baited container and the child 
twice, expressing her communicative intent through facial expressions (e.g., raised eyebrows and smile).

A different set of toys (presented in the same order across children) was used for each condition. Children were administered one of four quasi-random orders constructed by counterbalancing gesture within both the non-communicative and communicative conditions. However, the non-communicative trials (12 trials) were always presented before the communicative trials ( 12 trials) to avoid biasing the child's attention. ${ }^{1}$ For each of the four orders, the box in which the toy was hidden (left or right) was chosen randomly except for two restrictions: (1) A toy was never hidden more than twice in a row on the same side and (2) The toy was hidden three times on each side within each condition. If the child did not open the box containing the toy, the experimenter retrieved the toy for the child to encourage him/her to stay on task.

Coding-The communicative intent tasks were videotaped for coding purposes. Children's performance was coded based upon whether they successfully found the hidden toy during their initial approach toward the boxes. To evaluate the experimenter's adherence to the procedure, the coder, blind to the order of cue administration, recorded which of the four cues was observed and her confidence that the cue was demonstrated correctly. The primary coder coded all of the videotapes and indicated with a high level of confidence that for every trial, the correct cue was administered. To assess reliability, 20\% of the tapes for each diagnostic group (7 WS, 5 DS) were randomly selected and independently coded by a second person blind to all hypotheses of the study. The two coders agreed on $100 \%$ of the trials regarding which box the child had selected. In addition, the two coders also agreed on $100 \%$ of the trials regarding which of the 4 non-verbal cues the experimenter had presented, confirming that the experimenter had adhered to the procedure on all trials. No trials had to be excluded due to errors in administration.

\section{RESULTS}

Descriptive statistics for CA and Mullen standard scores are reported in Table 1.

Independent sample $t$-tests indicated the groups were not significantly different on CA [ $t(56)$ $=1.07, p=.29$ ] but that the children with DS $(M=52.48)$ had significantly lower Mullen composite DQs than the children with WS $(M=59.00)[t(56)=3.74, p<.001]$. The children with DS also had significantly lower raw scores than the children with WS on the Mullen Receptive Language $(p=.01)$ and Expressive Language $(p=.006)$ scales; the groups did not differ significantly on raw scores on the Visual Reception $(p=.35)$ and Fine Motor $(p=.81)$ scales.

As order of gesture was counterbalanced for both the non-communicative and communicative trials, analyses were computed to determine if order of pointing+ gazing and gazing only trials affected children's search performance. A one way ANOVA on the number of correct responses indicated no significant effect of order $[F(3,57)=0.31, p=$. 82]. Therefore, order was not included as a variable in any of the subsequent analyses.

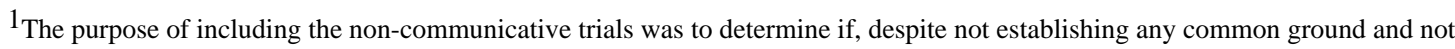
expressing any intent to show the child the location of the hidden toy, the simple presentation of an extended index finger or simply moving one's eyes to the location of the hidden toy was enough to direct the child's search performance. We felt that presenting the communicative trials prior to the non-communicative trials would establish a common ground between the adult and the child and that once the child understood that the adult was showing him/her the location of the hidden toy it would be impossible to retract this common ground.
} 


\section{Non-Communicative vs. Communicative Conditions: Comparisons to Chance}

We began by examining the responses of the WS and DS groups to determine if they were consistent with comprehension of the communicative intent behind the gestures or if they were consistent with use of low-level attentional cues. If children comprehended the communicative intent behind gestures, they would be expected to find the toy more often than expected by chance (chance $=6$ of 12 trials) on the communicative trials, while performance on the non-communicative trials would not be different from chance. In contrast, if children's search success is based on low-level attentional cueing processes rather than comprehending the communicative intent behind gestures they should find the toy more often than expected by chance on both the communicative trials and the noncommunicative trials. Results (see Figure 1) indicated that both the WS group $[t(32)=6.40$, $p<.001]$ and the DS group $[t(24)=7.08, p<.001]$ found the hidden toy significantly more often than expected by chance for the communicative trials. However, neither group found the toys significantly more than expected by chance on the non-communicative trials [WS: $t(32)=1.31, p=.20$; DS: $t(24)=0.40, p=.69$ ]. This pattern of findings indicates that, as groups, preschoolers with WS and preschoolers with DS were able to derive benefit from communicative non-linguistic gestures.

We then examined performance on the communicative trials relative to chance for each child to determine if the number of individual children who found the toy significantly more often than expected by chance differed as a function of diagnostic group. Based on the binomial distribution and a chance probability of success on each trial of .50, finding the toy on the first attempt for at least 10 out of 12 trials would indicate that the child performed significantly above the level expected by chance ( $p=.02$, one sided). Using this criterion, significantly more children with DS (60\%) than children with WS $(26.7 \%)$ found the toy more often than expected by chance $\left[\chi^{2}(1, N=58)=6.28 ; p=.01\right]$.

To explore the relations between performance on the communicative trials and CA and Mullen subscale raw scores, Pearson correlations were computed (Table 2). As WS and DS are associated with different genotypes, as well as different behavioral phenotypes, correlations were computed separately for each group. Differences were observed in factors related to overall communicative intent performance between children with WS and children with DS. For children with WS, the number of trials on which the child correctly inferred communicative intent as expressed through non-linguistic gestures was significantly related to raw scores on each of the four Mullen scales but not to CA. For children with DS, CA was significantly related to performance on the communicative intent task. Though correlations between raw scores on the Mullen Visual Reception $(p=.08)$ and Fine Motor $(p$ $=.07)$ scales and performance on the communicative intent task did not reach the conventional level of significance, they remain factors to consider in relation to comprehension of communicative intent.

\section{Communicative: Pointing + Gazing vs. Gazing only}

To examine possible differences in comprehension of the communicative intent behind pointing + gazing and gazing only gestures between children with WS or DS, a 2 (Gesture) $\times 2$ (Diagnostic Group) mixed ANOVA was calculated. Results indicated a significant main effect of Gesture $\left[F(1,56)=8.59, p=.005, \eta^{2}=.13\right]$. There also was a significant effect of Group $\left[F(1,56)=4.14, p=.047, \eta^{2}=.07\right]$ indicating that regardless of gesture type, children with DS were more likely than children with WS to find the hidden toy. The interaction term between Gesture and Diagnostic Group was not significant $\left[F(1,56)=0.65, p=.80, \eta^{2}=\right.$. 001]. As shown in Figure 2, both groups of children were able to derive benefit from communicative pointing + gazing and, to a lesser degree, communicative gazing only gestures. 
To determine the relation between children's performance in the communicative pointing + gazing and communicative gazing only conditions, Pearson correlations were calculated separately for the two groups of children. For the WS group, the correlation between performance in the two communicative conditions was not significant $(r=.19, p=.29)$. In contrast, the correlation was significant for the DS group $(r=.53, p=.007)$.

\section{DISCUSSION}

The ability to monitor and comprehend the communicative behaviors of others is critical to being able to function effectively in the social world (e.g., Akhtar \& Martinez-Sussmann, 2007; Bates et al., 1975; Carpenter et al., 1998; Hala, 1997; Moore \& Corkum, 1994). Although this ability emerges at an early age in most children, it requires at least rudimentary understanding of other people's underlying mental states (e.g., intentions, feelings, beliefs) and is therefore complex (e.g., Meltzoff, 1995; Thompson, 2006). In the case of gestures, understanding that intentions and goals underlie people's actions is vital as this realization allows a child not only to recognize that the action is informative but also to interpret why the person is doing what he/she is doing (Meltzoff, 1995). The aim of the present study was to evaluate this ability to comprehend the communicative intent behind pointing and gazing gestures by preschoolers who have WS or DS.

Our results indicate that at the group level, both preschoolers with WS and preschoolers with DS distinguish communicative intent from non-communicative intent. That is, children's search performance was influenced not by the experimenter's action itself but rather by the intention of that action. These findings show that at the group level, preschoolers with WS or DS are able to interpret whether an adult's nonverbal gestural behavior in a table-top object location task is a relevant communicative act directed toward them. However, at the individual child level, there were clear and significant differences in performance between children with DS and children with WS. In particular, whereas the majority of children with DS (60\%) followed communicative gestures to find the hidden object at a rate significantly above that expected by chance, only about a quarter of the children with WS (26.7\%) were able to do so. This finding of better gesture comprehension by preschoolers with DS than by preschoolers with WS is especially striking given that both mean DQ and mean raw scores on the Mullen Receptive Language and Expressive Language scales were significantly lower for the DS group than for the WS group. Both children with WS and children with DS had more difficulty inferring communicative intent from eye gaze alone than from eye gaze paired with a pointing gesture.

Although the present study did not include a TD control group, a general idea of how the performance of the WS and DS groups would compare to that of younger TD children may be obtained by considering the data from the participants in Behne et al. (2005), after which the present study was modeled. Behne and colleagues found that $84 \%$ of the 24 -month-olds in their sample performed significantly above chance on the communicative trials. For both syndromes included in the present study, the percentage of children who performed significantly above chance was strikingly lower. The percentage of children with DS who performed significantly above chance was the same as that found by Behne et al. for 18month-olds (60\%); the percentage of children with WS who performed significantly above chance was closest to that of the 14-months olds (7\%).

Nevertheless, all three groups showed the same pattern of difficulty in comprehension of communicative intent, with pointing + gazing gestures more likely to be comprehended than gazing only gestures. Several other researchers have reported findings supporting the hypothesis that, from a developmental perspective, children comprehend pointing gestures prior to gazing gestures (e.g., Aureli, Perucchini, \& Genco, 2009; Behne et al., 2005; Booth, 
McGregor, \& Rohlfing, 2008; Povinelli, Reaux, Bierschwale, Allain, \& Simon, 1997). The findings of the present study indicate that children with DS and children with WS demonstrate the same developmental progression regarding comprehension of pointing + gazing gestures and gazing only gestures as has been found for TD children, although both syndrome groups evidenced significant delays.

Our findings for children with WS or DS, combined with those from previous studies of TD toddlers, demonstrate a developmental progression regarding comprehension of pointing + gazing gestures and gazing only gestures. These findings raise the question of why children comprehend pointing+ gazing gestures earlier in development than they comprehend gazing gestures. It is likely that multiple factors contribute to the discrepancy between success at gaze following and success at point following shown by young children. For example, as noted by Aureli and colleagues (2009), although adults commonly use pointing paired with gaze shifting to express communicative intent when interacting with young children, gaze shifting alone is not typically used for this purpose. As such, children's unfamiliarity with gazing only gestures contributes to poorer performance in conditions that depend on gaze following either because the children do not comprehend the meaning of the experimenter's gaze or perhaps because even if they did realize that the experimenter was intending to communicate with them, they could not locate the target. Similarly, Booth and colleagues (2008) argued that the gestures that are most specifically associated with communicating referential intent are most likely to cue the child's recognition of communicative intent. As pointing paired with gaze shifting is used almost exclusively to indicate an object whereas gaze shifting only is used for multiple purposes, pointing should be more indicative of referential communicative intent. Booth and colleagues (2008) also discussed the role of attentional cueing in the comprehension of gestures, arguing that cues that are more salient and that demonstrate a clearer path between speaker and referent are easier to follow. On these grounds as well, pointing paired with gaze shifting once again would be considered a richer communicative signal (with duplication of referential information in two gesture modalities) and easier to follow than gaze shifting alone.

The results of the present study, the first to examine the ability of children with WS or DS to comprehend the communicative intent behind gestures, are consistent with previous findings regarding the overall socio-communicative abilities of individuals with these syndromes. Although children with WS demonstrate relative strengths in language and appear to be interested in people (Mervis \& Klein-Tasman, 2000; Mervis \& Morris, 2007), they demonstrate significant difficulties interacting with people and significant impairments in pragmatics (for review see Mervis \& Becerra, 2007; Tager-Flusberg \& Plesa Skwerer, 2006). The socio-communicative phenotype for children with DS appears to be very different. Despite a relative weakness in expressive language, children with DS show relative strengths in nonverbal communication (Miller \& Leddy, 1999) and social emotional skills (Fidler, 2005; Kasari, 2002). The present results extend these findings by demonstrating a similar pattern of performance inability to recognize the communicative intent behind gestures. Despite significantly lower mean DQ and language levels, the DS group was significantly better than the WS group at understanding when the experimenter's gesture was meant to relate to the ongoing interaction shared between the child and the experimenter. This pattern of findings provides further evidence of socio-communicative difficulties for children with WS and relative strength in socio-communicative abilities for children with DS.

\section{Limitations/Future Directions}

We must acknowledge two limitations of this study. First, the video cameras were not positioned close enough to the children to allow for joint attention to be coded. Thus, we were not able to objectively test the experimenters' anecdotal reports that whereas most of 
the children with DS were actively using the experimenter to find the location of the hidden toy; most of the children with WS were not. We hypothesize that after being presented with the communicative gesture, the children who understood the premise of the task engaged in joint attention episodes with the experimenter to confirm the information communicated. In future studies, we recommend that the cameras be positioned so that it is possible to directly code the children's nonverbal behaviors in response to communicative gestures to provide more information regarding their behavior when communicative intent was inferred versus when communicative intent was not inferred. Second, we only included six trials in each condition. Ideally, we would have included more trials so that we could have examined performance separately for communicative pointing and communicative gazing relative to chance. However, during pilot testing we found that including eight trials in all four conditions made the task long enough that many children lost interest in the game. Using a between subjects design would allow for the inclusion of more trials in each condition. However, such a design would require a considerable increase in the number of participants, which likely would be impractical given the rarity of WS. To document the developmental trajectories of the ability of children with WS and children with DS to follow a communicative point or a communicative gaze, longitudinal studies would be particularly useful.

\section{Conclusions}

As groups, preschoolers with WS or DS were able to distinguish communicative intent from non-communicative intent for pointing paired with gazing and gazing only gestures in the context of a table-top object location task. Despite significantly lower DQs and receptive and expressive language levels, the DS group evidenced significantly stronger pragmatic skills than the WS group. This finding provides further evidence supporting the sociocommunicative profiles associated with these syndromes. Although children with WS have a relative strength in language and are gregarious, they have a clear weakness in pragmatics, including nonverbal communication. In particular, our findings indicate that, in the context of our task, children with WS did not reliably interpret an adult's nonverbal communicative gesture as a relevant communicative act directed toward them intended to influence their behavior. In contrast, although children with DS have a relative weakness in expressive language, they have a relative strength in pragmatics, including nonverbal communication, and were much more likely to appropriately interpret a nonverbal gesture directed toward them. This pattern of findings provides further evidence that young children with WS have more difficulty with socio-communication than expected for either CA or level of intellectual or language ability. This pattern also offers suggestions regarding syndromespecific early intervention strategies: Given the socio-communicative weaknesses evidenced by children with WS, it is important that interventions be developed to target these areas directly and that providers not be deceived by the children's relatively good expressive language into thinking that their communicative skills in general are adequate for their developmental level, as has happened all too often in the past. For children with DS, in addition to focusing on expressive language development, intervention programs should be designed to use the children's relative strength in socio-communication to help them compensate for difficulties with expressive language.

\section{Acknowledgments}

This research was supported by grants R37 HD29957 from the National Institute of Child Health and Human Development and R01 NS35102 from the National Institute of Neurological Disorders and Stroke. We thank the children and their parents for their enthusiastic participation in our research. Melissa Rowe and Alicia Barber assisted with data collection. 


\section{References}

Adamson, LB. Communication development during infancy. Madison, WI: Brown and Benchmark; 1995.

Akhtar, N.; Martinez-Sussmann, C. Intentional communication. In: Brownell, CA.; Kopp, CB., editors. Socioemotional development in the toddler years: Transitions and transformations. New York, NY: Guilford Press; 2007. p. 201-220.

Aureli T, Perucchini P, Genco M. Children's understanding of communicative intentions in the middle of the second year of life. Cognitive Development 2009;24:1-12.

Bates E, Camaioni L, Volterra V. The acquisition of performatives prior to speech. Merrill Palmer Quarterly 1975;21:205-226.

Behne T, Carpenter M, Tomasello M. One-year-olds comprehend the communicative intentions behind gestures in a hiding game. Developmental Science 2005;8:492-499. [PubMed: 16246240]

Booth AE, McGregor KK, Rohlfing KJ. Socio-pragmatics and attention: Contributions to gesturally guided word learning in toddlers. Language Learning and Development 2008;4:179-202.

Brooks R, Meltzoff AN. The importance of the eyes: How infants interpret adult looking behavior. Developmental Psychology 2002;38:958-966. [PubMed: 12428707]

Butterworth, G. Joint visual attention in infancy. In: Bremner, G.; Fogel, A., editors. Blackwell handbook of infant development. Oxford, UK: Blackwell; 2001. p. 213-240.

Butterworth, G. Pointing is the royal road to language for babies. In: Kita, S., editor. Pointing: Where language, culture, and cognition meet. Mahwah, NJ: Erlbaum; 2003. p. 9-33.

Call, J.; Tomasello, M. What do chimpanzees know about seeing revisited: An explanation of the third kind. In: Eilan, N.; Hoerl, C.; McCormack, T.; Roessler, J., editors. Issues in joint attention. Oxford, England: Oxford University Press; 2005. p. 45-64.

Carpenter M, Nagell K, Tomasello M. Social cognition, joint attention, and communicative competence from 9 to 15 months of age. Monographs of the Society for Research in Child Development 1998;63(4 No 255)

Centers for Disease Control. Improved national prevalence estimates for 18 selected major birth defects -United States, 1999-2001. Morbidity and Mortality Weekly Report 2006;54(51\&52): 1301-1305. [PubMed: 16397457]

Chapman, RS. Language development in children and adolescents with Down syndrome. In: Miller, JF.; Leddy, M.; Leavitt, LA., editors. Improving the communication of people with Down syndrome. Baltimore, MD: Brookes; 1999. p. 81-92.

Corkum, V.; Moore, C. Development of joint visual attention in infants. In: Moore, C.; Dunham, PJ., editors. Joint attention: Its origins and role in development. Hillsdale, NJ: Erlbaum; 1995. p. 61-83.

D'Entremont B, Hains SMJ, Muir DW. A demonstration of gaze following in 3-to 6- month-olds. Infant Behavior and Development 1997;20:569-572.

Dilts CV, Morris CA, Leonard CO. Hypothesis for development of a behavioral phenotype in Williams syndrome. American Journal of Medical Genetics Supplement 1990;6:126-131. [PubMed: 2118772]

Dykens EM, Rosner BA. Refining behavioral phenotypes: Personality-motivation in Williams and Prader-Willi syndromes. American Journal on Mental Retardation 1999;104:158-169. [PubMed: 10207579]

Edgin, JO. Doctoral dissertation. 2003. A neuropsychological model for the development of the cognitive profiles in mental retardation syndromes: Evidence from Down syndrome and Williams syndrome. Retrieved from ProQuest. (AAT 3086381)

Ewart AK, Morris CA, Atkinson D, Jin W, Sternes K, Spallone P, et al. Hemizygosity at the elastin locus in a developmental disorder, Williams syndrome. Nature Genetics 1993;5:11-16. [PubMed: 7693128]

Fidler DJ. The emerging Down syndrome behavioral phenotype in early childhood: Implications for practice. Infants and Young Children 2005;18:86-103.

Fryns J, Borghgraef M, Volcke P, van den Berge H. Adults with Williams syndrome. American Journal of Medical Genetics 1991;40:253. [PubMed: 1897583]

J Speech Lang Hear Res. Author manuscript; available in PMC 2011 August 1. 
Gosch A, Pankau R. Personality characteristics and behaviour problems in individuals of different ages with Williams syndrome. Developmental Medicine \& Child Neurology 1997;39:527-533. [PubMed: 9295848]

Hala, S., editor. The development of social cognition. Hove, England: Psychology Press; 1997.

Kasari C. Assessing change in early intervention programs for children with autism. Journal of Autism and Developmental Disorders 2002;32:447-461. [PubMed: 12463519]

Kasari C, Freeman SFN, Bass W. Empathy and response to distress in children with Down syndrome. Journal of Child Psychology \& Psychiatry 2003;44:424-431. [PubMed: 12635971]

Klein BP, Mervis CB. Cognitive strengths and weaknesses of 9-and 10-year-olds with Williams syndrome or Down syndrome. Developmental Neuropsychology 1999;16:177-196.

Klein-Tasman BP, Mervis CB, Lord C, Phillips K. Socio-communicative deficits in young children with Williams syndrome: Performance on the Autism Diagnostic Observation Schedule. Child Neuropsychology 2007;13:444-467. [PubMed: 17805996]

Laing E, Butterworth G, Ansari D, Gsodl M, Longhi E, Panagiotaki G, et al. Atypical development of language and social communication in toddlers with Williams syndrome. Developmental Science 2002;5:233-246.

Lincoln AJ, Searcy YM, Jones W, Lord C. Social interaction behaviors discriminate young children with autism and Williams syndrome. Journal of the American Academy of Child \& Adolescent Psychiatry 2007;46:323-331. [PubMed: 17314718]

Lord, C.; Rutter, M.; DiLavore, PC.; Risi, S. Autism diagnostic observation schedule -WPS edition. Los Angeles, CA: Western Psychological Services; 1999.

Meltzoff AN. Understanding the intentions of others. Re-enactment of intended acts by 18 -month-old children. Developmental Psychology 1995;31:838-850.

Mervis CB, Becerra AM. Language and communicative development in Williams syndrome. Mental Retardation and Developmental Disabilities Research Reviews 2007;13:3-15. [PubMed: 17326109]

Mervis CB, Klein-Tasman BP. Williams syndrome: Cognition, personality, and adaptive behavior. Mental Retardation and Developmental Disabilities Research Reviews 2000;6:148-158. [PubMed: 10899809]

Mervis, CB.; Morris, CA. Williams syndrome. In: Mazzocco, MMM.; Ross, JL., editors. Neurogenetic developmental disorders: Variation of manifestation in childhood. Cambridge, MA: MIT Press; 2007. p. 199-262.

Miller, JF.; Leddy, M. Verbal fluency, speech intelligibility, and communicative effectiveness. In: Miller, JF.; Leddy, M.; Leavitt, LA., editors. Improving the communication of people with Down syndrome. Baltimore, MD: Brookes; 1999. p. 81-97.

Moore C, Corkum V. Social understanding at the end of the first year of life. Developmental Review 1994;14:349-372.

Morris, CA. The dysmorphology, genetics, and natural history of Williams-Beuren syndrome. In: Morris, CA.; Lenhoff, HM.; Wang, PP., editors. Williams-Beuren syndrome: Research, evaluation and treatment. Baltimore, MD: The Johns Hopkins University Press; 2006. p. 3-17.

Mundy, P.; Hogan, A. A preliminary manual for the abridged Early Social Communication Scales (ECSC). Available through the University of Miami Psychological Department; Coral Gables, FL: 1996. http://www.psy.miami.edu/faculty/pmundy/ESCS.pdf

Povinelli DJ, Reaux JE, Bierschwale DT, Allain AD, Simon BB. Exploitation of pointing as a referential gesture in young children, but not adolescent chimpanzees. Cognitive Development 1997;12:327-365.

Rowe, ML. Doctoral dissertation. 2007. Mastery motivation in young children with Williams syndrome or Down syndrome. Retrieved from ProQuest. (AAT 3267110)

Rowe, ML.; Peregrine, E.; Mervis, CB. Communicative development in toddlers with Williams syndrome. Poster presented at the Society for Research in Child Development; Atlanta, GA. 2005 Apr.

Sigman M, Ruskin E. Continuity and change in the social competence of children with autism, Down syndrome, and developmental delays. Monographs of the Society for Research in Child Development 1999;64(1 No 256) 
Strømme P, Bjørnstad PG, Ramstad K. Prevalence estimation of Williams syndrome. Journal of Child Neuropsychology 2002;17:269-271.

Tager-Flusberg, H.; Plesa Skwerer, D. Social engagement in Williams syndrome. In: Marshall, PJ.; Fox, N., editors. The development of social engagement: Neurobiological perspectives. Oxford, England: Oxford University Press; 2006. p. 331-354.

Thompson, RA. The development of the person: Social understanding, relationships, conscience, and self. In: Eisenberg, N.; Damon, W.; Lerner, RM., editors. Handbook of child psychology. 6. Vol. 3. Hoboken, NJ: Wiley; 2006. p. 24-98.

Tomasello M, Call J, Gluckman A. Comprehension of novel communicative signs by apes and human children. Child Development 1997;68:1067-1080. [PubMed: 9418226]

Tomasello M, Carpenter M, Liszkowski U. A new look at infant pointing. Child Development 2007;78:705-722. [PubMed: 17516997]

Udwin O, Yule W. A cognitive and behavioral phenotype in Williams syndrome. Journal of Clinical and Experimental Neuropsychology 1990;13:232-244. [PubMed: 1864913]

J Speech Lang Hear Res. Author manuscript; available in PMC 2011 August 1. 


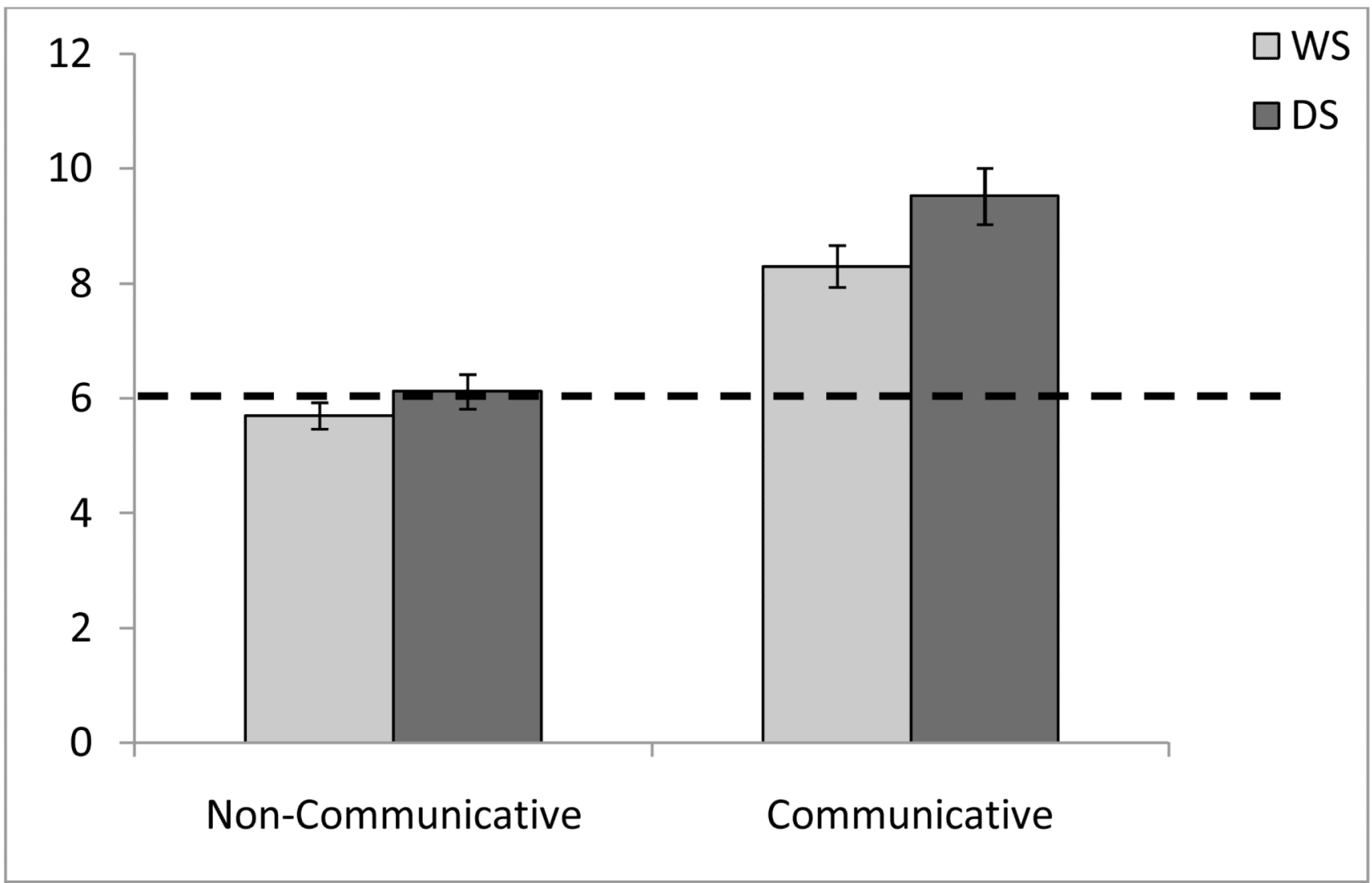

Figure 1.

Number of trials correct on the non-communicative vs. communicative trials relative to chance as a function of diagnostic group. Error bars represent standard error of the mean and dotted line indicates chance level of performance. WS = Williams syndrome; DS = Down syndrome. 


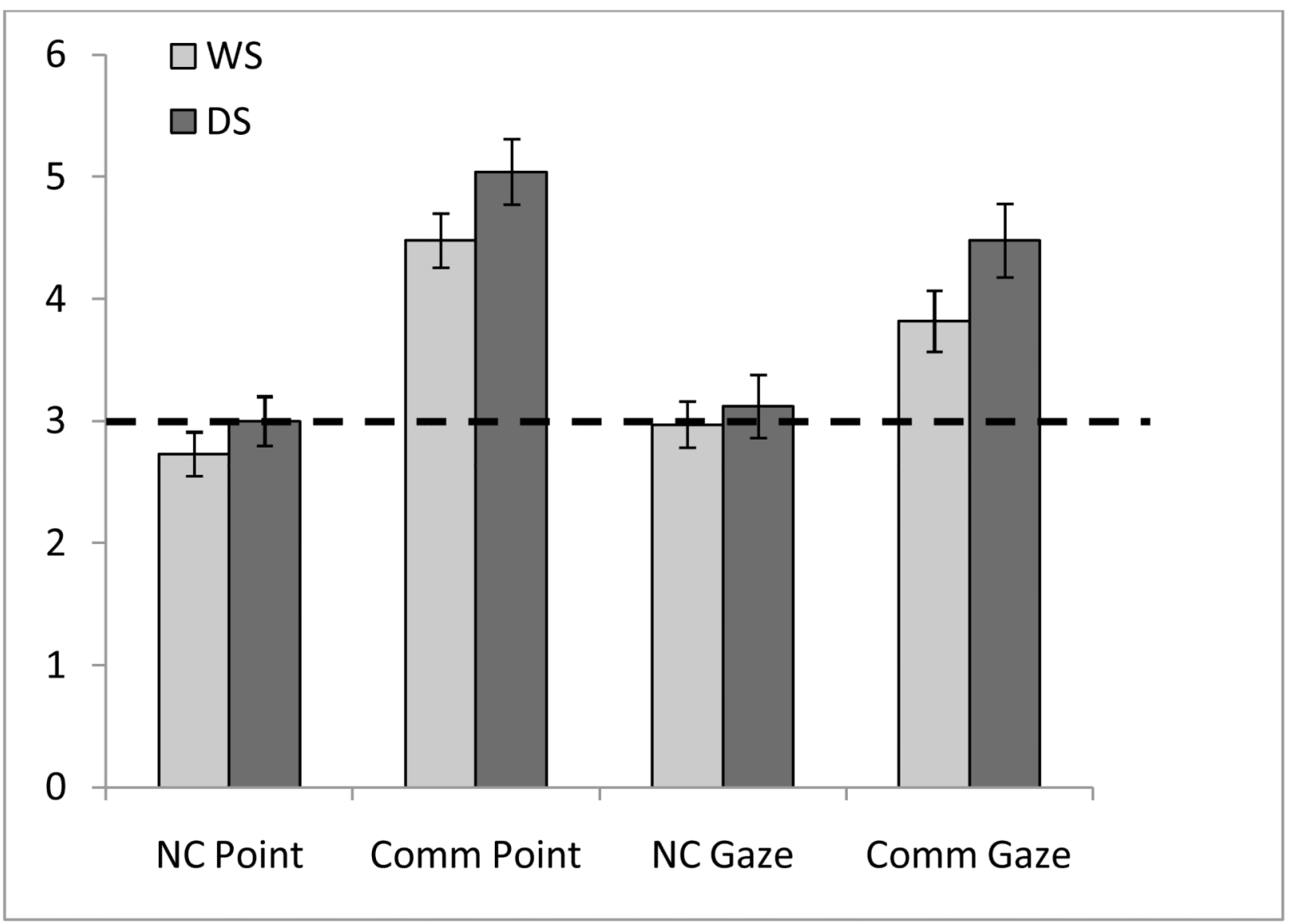

Figure 2.

Number of trials correct for each condition relative to chance performance as a function of diagnostic group. Error bars represent standard error of the mean and dotted line indicates chance level of performance. WS $=$ Williams syndrome; DS = Down syndrome $; \mathrm{NC}=$ Noncommunicative; Comm $=$ Communicative. 


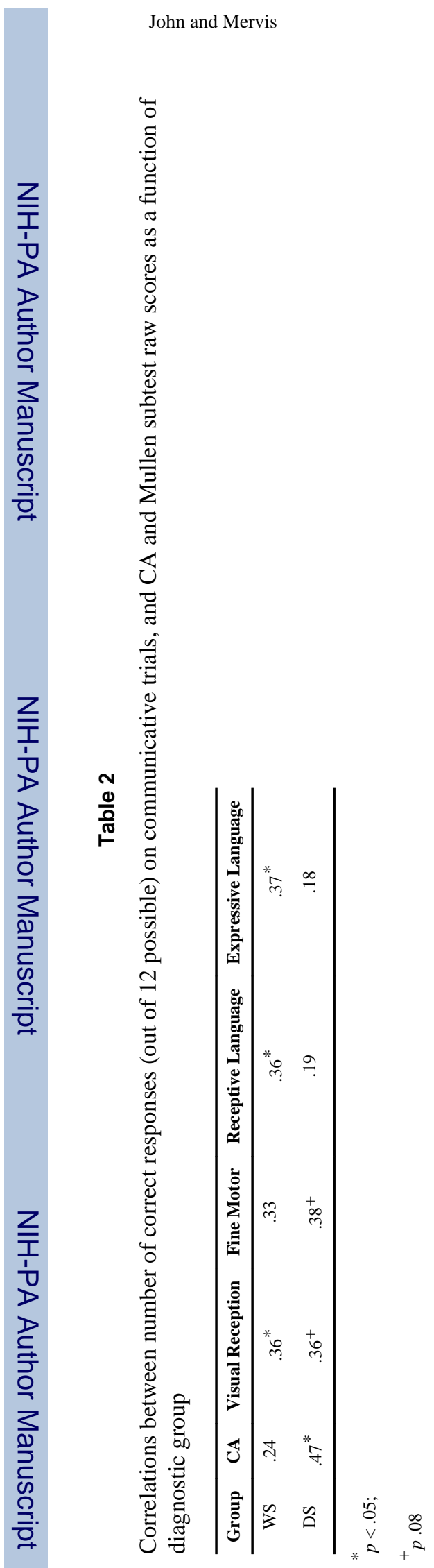

J Speech Lang Hear Res. Author manuscript; available in PMC 2011 August 1. 